\title{
Research Article \\ Propagation of Computer Virus under Human Intervention: A Dynamical Model
}

\author{
Chenquan Gan, ${ }^{1,2}$ Xiaofan Yang, ${ }^{1,2}$ Wanping Liu, ${ }^{1}$ \\ Qingyi Zhu, ${ }^{1}$ and Xulong Zhang ${ }^{1}$ \\ ${ }^{1}$ College of Computer Science, Chongqing University, Chongqing 400044, China \\ ${ }^{2}$ School of Electronic and Information Engineering, Southwest University, Chongqing 400715, China \\ Correspondence should be addressed to Xiaofan Yang, xf_yang1964@yahoo.com
}

Received 13 May 2012; Accepted 7 June 2012

Academic Editor: Yanbing Liu

Copyright (c) 2012 Chenquan Gan et al. This is an open access article distributed under the Creative Commons Attribution License, which permits unrestricted use, distribution, and reproduction in any medium, provided the original work is properly cited.

\begin{abstract}
This paper examines the propagation behavior of computer virus under human intervention. A dynamical model describing the spread of computer virus, under which a susceptible computer can become recovered directly and an infected computer can become susceptible directly, is proposed. Through a qualitative analysis of this model, it is found that the virus-free equilibrium is globally asymptotically stable when the basic reproduction number $R_{0} \leq 1$, whereas the viral equilibrium is globally asymptotically stable if $R_{0}>1$. Based on these results and a parameter analysis, some appropriate measures for eradicating the spread of computer virus across the Internet are recommended.
\end{abstract}

\section{Introduction}

Due to their striking features such as destruction, polymorphism, and unpredictability [1, 2], computer viruses have come to be one major threat to our work and daily life $[3,4]$. With the rapid advance of computer and communication technologies, computer virus programs are becoming increasingly sophisticated so that developing antivirus software is becoming increasingly expensive and time-consuming [5]. Dynamical modeling of the spread process of computer virus is an effective approach to the understanding of behavior of computer viruses because, on this basis, some effective measures can be posed to prevent infection. In the past decade or so, a number of epidemic models (SEIR model [6, 7], SEIRS model [8], SIRS model [9-15], SEIQV model [16], SEIQRS model [17] and SAIR model [4], were simply borrowed to depict the spread of computer virus.

In reality, human intervention plays an important role in slowing down the propagation of computer viruses or preventing the breakout of computer viruses, under which a susceptible computer can become recovered directly, and an infected computer can become 
susceptible directly. To our knowledge, however, all the previous models did not consider the effect of human intervention.

In this paper, a new computer virus propagation model, which incorporates the above mentioned effects of human intervention, is proposed. The dynamics of this model are investigated. Specifically, the virus-free equilibrium is globally asymptotically stable when the basic reproduction number $R_{0} \leq 1$, whereas the viral equilibrium is globally asymptotically stable if $R_{0}>1$. Based on these results and a parameter analysis, some effective strategies for eradicating computer viruses are advised.

The subsequent materials of this paper are organized as follows: Section 2 formulates the model; Section 3 shows the global stability of the virus-free equilibrium; Section 4 proves the global stability of the viral equilibrium; Some policies are posed in Section 5 for controlling virus spread; finally, this work is summarized in Section 6.

\section{Assumptions and Model Formulation}

At any given time, computers all over the world are classified as internal or external depending on whether it is currently accessing to the Internet or not, and all internal computers are further categorized into three classes: (1) susceptible computers, that is, virus-free computers having no immunity; (2) infected computers; (3) recovered computers, that is, virus-free computers having immunity. At time $t$, let $S(t), I(t)$, and $R(t)$ denote the concentrations (i.e., percentages) of susceptible, infected, and recovered computers in all internal computers, respectively. Then $S(t)+I(t)+R(t) \equiv 1$. Without ambiguity, $S(t), I(t)$, and $R(t)$ will be abbreviated as $S, I$, and $R$, respectively.

Our model is based on the following assumptions.

(A1) All newly accessed computers are virus-free. Furthermore, due to the effect of newly accessed computers, at any time the percentage of susceptible computers increases by $\delta$.

(A2) At any time an internal computer is disconnected from the Internet with probability $\delta$.

(A3) Due to the effect of previously infected computers, at any time the percentage of infected computers increases by $\beta S I$, where $\beta$ is a positive constant.

(A4) Due to the effect of cure, at any time an infected computer becomes recovered with probability $\gamma_{1}$, or becomes susceptible with probability $\gamma_{2}$.

(A5) Due to the loss of immunity, at any time a recovered computer becomes susceptible with probability $\alpha_{2}$.

(A6) Due to the availability of new vaccine, at any time a susceptible computer becomes recovered with probability $\alpha_{1} I$.

This collection of assumptions can be schematically shown in Figure 1, from which one can derive the following model describing the propagation of computer virus:

$$
\begin{gathered}
\dot{S}=\delta-\alpha_{1} S I-\delta S+\gamma_{2} I-\beta S I+\alpha_{2} R, \\
\dot{I}=\beta S I-\gamma_{2} I-\delta I-\gamma_{1} I, \\
\dot{R}=\gamma_{1} I+\alpha_{1} S I-\delta R-\alpha_{2} R,
\end{gathered}
$$

with initial conditions $S(0) \geq 0, I(0) \geq 0$ and $R(0) \geq 0$. 


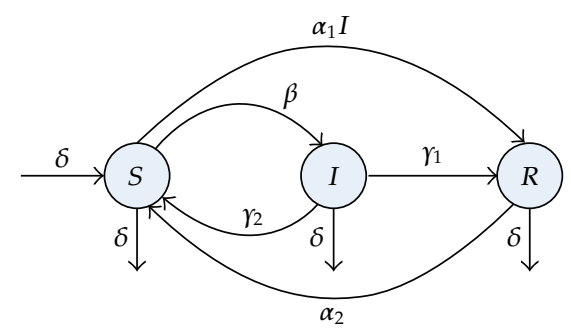

Figure 1: The transfer diagram of the SIRS model.

The basic reproduction number, $R_{0}$, is defined as the average number of susceptible computers that are infected by a single infected computer during its life span. From the above model, one can derive the basic reproduction number $R_{0}$ as

$$
R_{0}=\frac{\beta}{\gamma_{1}+\gamma_{2}+\delta}
$$

Because $S+I+R \equiv 1$, system (2.1) simplifies to the following planar system:

$$
\begin{aligned}
& \dot{S}=\delta-\alpha_{1} S I-\delta S+\gamma_{2} I-\beta S I+\alpha_{2}(1-S-I), \\
& \dot{I}=\beta S I-\gamma_{2} I-\delta I-\gamma_{1} I,
\end{aligned}
$$

with initial conditions $S(0) \geq 0$ and $I(0) \geq 0$. Clearly, the feasible region for this system is $\Omega=\{(S, I): S \geq 0, I \geq 0, S+I \leq 1\}$, which is positively invariant.

\section{The Virus-Free Equilibrium and Its Stability}

System (2.3) always has a virus-free equilibrium $E^{0}(1,0)$. Next, let us consider its global stability by means of the Direct Lyapunov Method.

Theorem 3.1. $E^{0}$ is globally asymptotically stable with respect to $\Omega$ if $R_{0} \leq 1$.

Proof. Let $V(t)=I(t)$, then

$$
\begin{aligned}
\left.V^{\prime}(t)\right|_{(2.3)}=\dot{I} & =\beta S I-\gamma_{2} I-\delta I-\gamma_{1} I \\
& =\beta I\left(S-\frac{\gamma_{1}+\gamma_{2}+\delta}{\beta}\right) \\
& =\beta I\left(S-\frac{1}{R_{0}}\right) .
\end{aligned}
$$

Because $R_{0} \leq 1$ and $S+I \leq 1$, we have $\left.V^{\prime}(t)\right|_{(3)} \leq 0$. Moreover, $\left.V^{\prime}(t)\right|_{(3)}=0$ if and only if $(S, I)=(1,0)$. Thus, the claimed result follows from the LaSalle Invariance Principle. 


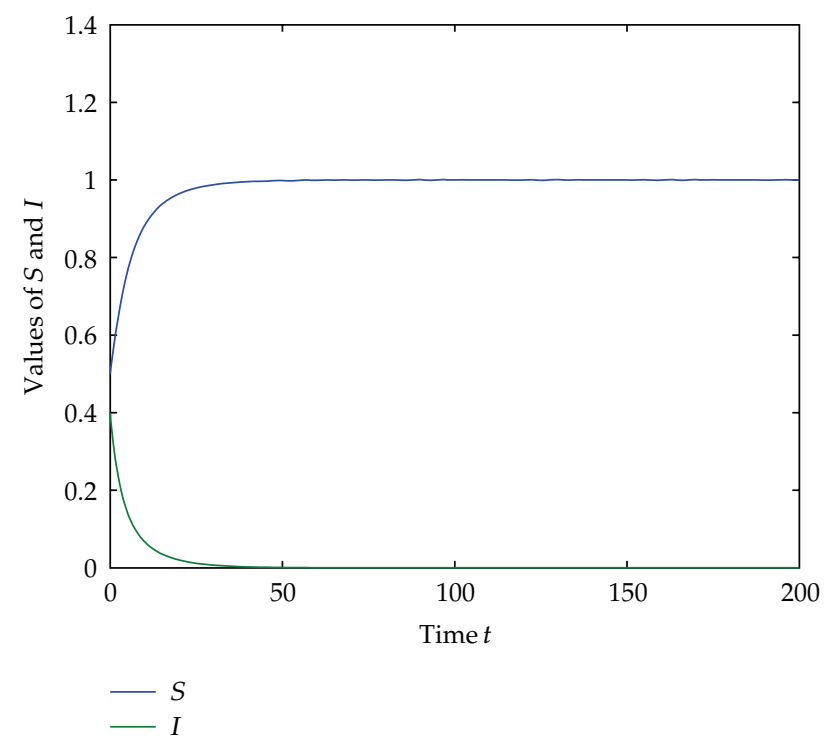

Figure 2: Evolutions of $S(t)$ and $I(t)$ for the system with $\beta=0.3, \delta=0.1, \alpha_{1}=0.2, \alpha_{2}=0.4, \gamma_{1}=0.1$ and $\gamma_{2}=0.2$, provided $S(0)=0.5$ and $I(0)=0.4$.

Example 3.2. Consider a system of the form (2.3) and with $\beta=0.3, \delta=0.1, \alpha_{1}=0.2, \alpha_{2}=0.4$, $\gamma_{1}=0.1$, and $\gamma_{2}=0.2$. Then $R_{0}=0.75<1$. By Theorem 3.1, the virus-free equilibrium is globally asymptotically stable. Figure 2 demonstrates how $S(t)$ and $I(t)$ evolve with time $t$ if $S(0)=0.5$ and $I(0)=0.4$.

\section{The Viral Equilibrium and Its Stability}

When $R_{0}>1$, it is easy to verify that system (2.3) has a unique viral equilibrium $E^{*}\left(S^{*}, I^{*}\right)$, where

$$
S^{*}=\frac{\delta+\gamma_{1}+\gamma_{2}}{\beta}=\frac{1}{R_{0}}, \quad I^{*}=\frac{\left(\delta+\alpha_{2}\right)\left(R_{0}-1\right)}{\alpha_{1}+\left(\delta+\gamma_{1}+\alpha_{2}\right) R_{0}}>0
$$

First, consider the local stability of $E^{*}$.

Theorem 4.1. $E^{*}$ is locally asymptotically stable if $R_{0}>1$.

Proof. For the linearized system of system (2.3) at $E^{*}$, the corresponding Jacobian matrix is

$$
J_{E^{*}}=\left(\begin{array}{cc}
-\alpha_{2}-\delta-\left(\alpha_{1}+\beta\right) I^{*} & -\left(\alpha_{2}+\gamma_{1}+\delta+\alpha_{1} S^{*}\right) \\
\beta I^{*} & 0
\end{array}\right) .
$$

The characteristic equation of $J_{E^{*}}$ is

$$
\lambda^{2}+k_{1} \lambda+k_{2}=0
$$


where

$$
\begin{aligned}
& k_{1}=\left(\alpha_{1}+\beta\right) I^{*}+\alpha_{2}+\delta>0, \\
& k_{2}=\left(\alpha_{2}+\gamma_{1}+\delta+\alpha_{1} S^{*}\right) \beta I^{*}>0 .
\end{aligned}
$$

It follows from the Hurwitz criterion that the two roots of (4.3) have negative real parts. Hence, the claimed result follows.

We are ready to study global stability of $E^{*}$. Let $\Omega^{\prime}=\Omega-E^{0}$, then we have as following.

Theorem 4.2. $E^{*}$ is globally asymptotically stable with respect to $\Omega^{\prime}$ if $R_{0}>1$.

Proof. From system (2.3), we have

$$
\delta-\alpha_{1} S^{*} I^{*}-\delta S^{*}+\gamma_{2} I^{*}-\beta S^{*} I^{*}+\alpha_{2}\left(1-S^{*}-I^{*}\right)=0 .
$$

Note that

$$
\frac{1}{\beta}\left(\frac{\alpha_{2}-\gamma_{2}}{S^{*}}+\alpha_{1}\right)+1=\frac{1}{\beta}\left(\frac{\alpha_{2}+\gamma_{1}+\delta}{S^{*}}+\alpha_{1}\right)>0
$$

Define the Lyapunov function as

$$
V(t)=\int_{S^{*}}^{S} \frac{x-S^{*}}{x} d x+(d+1) \int_{I^{*}}^{I} \frac{x-I^{*}}{x} d x
$$

where $d=(1 / \beta)\left(\left(\alpha_{2}-\gamma_{2}\right) / S^{*}+\alpha_{1}\right)$. Then

$$
\begin{aligned}
\left.V^{\prime}(t)\right|_{(2.3)}= & \left(1-\frac{S^{*}}{S}\right) \dot{S}+(d+1)\left(1-\frac{I^{*}}{I}\right) \dot{I} \\
= & \left(1-\frac{S^{*}}{S}\right)\left[\delta-\alpha_{1} S I-\delta S+\gamma_{2} I-\beta S I+\alpha_{2}(1-S-I)\right] \\
& +(d+1)\left(1-\frac{I^{*}}{I}\right)\left(\beta S I-\gamma_{2} I-\delta I-\gamma_{1} I\right) \\
= & \left(1-\frac{S^{*}}{S}\right)\left[\delta-\alpha_{1} S I-\delta S+\gamma_{2} I-\beta S I+\alpha_{2}(1-S-I)\right] \\
& +(d+1)\left(1-\frac{I^{*}}{I}\right)\left(1-\frac{S^{*}}{S}\right) \beta S I
\end{aligned}
$$




$$
\begin{aligned}
& =\left(1-\frac{S^{*}}{S}\right)\left[\alpha_{1} S^{*} I^{*}-\alpha_{1} S I+\delta\left(S^{*}-S\right)+\gamma_{2}\left(I-I^{*}\right)\right. \\
& \left.+\alpha_{2}\left(S^{*}-S\right)+\alpha_{2}\left(I^{*}-I\right)+\beta d S I+\beta d S^{*} I^{*}-\beta(d+1) S I^{*}\right] \\
& =\left(1-\frac{S^{*}}{S}\right)\left[\left(S^{*}-S\right)\left(\alpha_{1} I^{*}+\delta+\alpha_{2}+\beta I^{*}\right)+\left(I-I^{*}\right)\left(\beta d-\alpha_{1}\right)\left(S-\frac{\alpha_{2}-\gamma_{2}}{\beta d-\alpha_{1}}\right)\right] \\
& =-\frac{\left(S^{*}-S\right)^{2}}{S}\left(\delta+\alpha_{2}+\beta I^{*}-\frac{\alpha_{2}-\gamma_{2}}{S^{*}} I+\frac{\alpha_{2}-\gamma_{2}}{S^{*}} I^{*}+\alpha_{1} I^{*}\right) .
\end{aligned}
$$

If $\alpha_{2} \leq \gamma_{2}$, because $\beta I^{*}+\left(\left(\alpha_{2}-\gamma_{2}\right) / S^{*}\right) I^{*}=\left(\left(\alpha_{2}+\gamma_{1}+\delta\right) / S^{*}\right) I^{*}>0$, thus

$$
\delta+\alpha_{2}+\beta I^{*}-\frac{\alpha_{2}-\gamma_{2}}{S^{*}} I+\frac{\alpha_{2}-\gamma_{2}}{S^{*}} I^{*}+\alpha_{1} I^{*}>0
$$

If $\alpha_{2}>\gamma_{2}$, from (4.5) we get

$$
\begin{aligned}
(\delta+ & \left.\alpha_{2}+\beta I^{*}+\frac{\alpha_{2}-\gamma_{2}}{S^{*}} I^{*}+\alpha_{1} I^{*}\right) \frac{S^{*}}{\alpha_{2}-\gamma_{2}} \\
= & \left(\delta+\alpha_{2}+\beta I^{*}+\frac{\alpha_{2}-\gamma_{2}}{S^{*}} I^{*}+\alpha_{1} I^{*}\right) \frac{S^{*}}{\alpha_{2}-\gamma_{2}} \\
& +\left[\delta-\alpha_{1} S^{*} I^{*}-\delta S^{*}+\gamma_{2} I^{*}-\beta S^{*} I^{*}+\alpha_{2}\left(1-S^{*}-I^{*}\right)\right] \frac{1}{\alpha_{2}-\gamma_{2}} \\
= & \frac{\delta+\alpha_{2}}{\alpha_{2}-\gamma_{2}}>1>I .
\end{aligned}
$$

Hence, we have

$$
\delta+\alpha_{2}+\beta I^{*}-\frac{\alpha_{2}-\gamma_{2}}{S^{*}} I+\frac{\alpha_{2}-\gamma_{2}}{S^{*}} I^{*}+\alpha_{1} I^{*}>0
$$

Furthermore, it is easy to see that $\left.V^{\prime}(t)\right|_{(2.3)} \leq 0$, and $\left.V^{\prime}(t)\right|_{(2.3)}=0$ if and only if $(S, I)=\left(S^{*}, I^{*}\right)$. Hence, the claimed result follows from the LaSalle invariance principle.

Example 4.3. Consider a system of the form (2.3) and with $\beta=0.3, \delta=0.1, \alpha_{1}=0.2, \alpha_{2}=0.4$, $\gamma_{1}=0.1$, and $\gamma_{2}=0.05$. Then $R_{0}=1.2>1$. It follows from Theorem 4.2 that the viral equilibrium is globally asymptotically stable. Figure 3 displays how $S(t)$ and $I(t)$ evolve with time $t$ if $S(0)=0.5$ and $I(0)=0.4$.

\section{Discussions}

As was indicated in the previous two sections, in order to eradicate computer viruses, one should take actions to keep $R_{0}$ below one. 


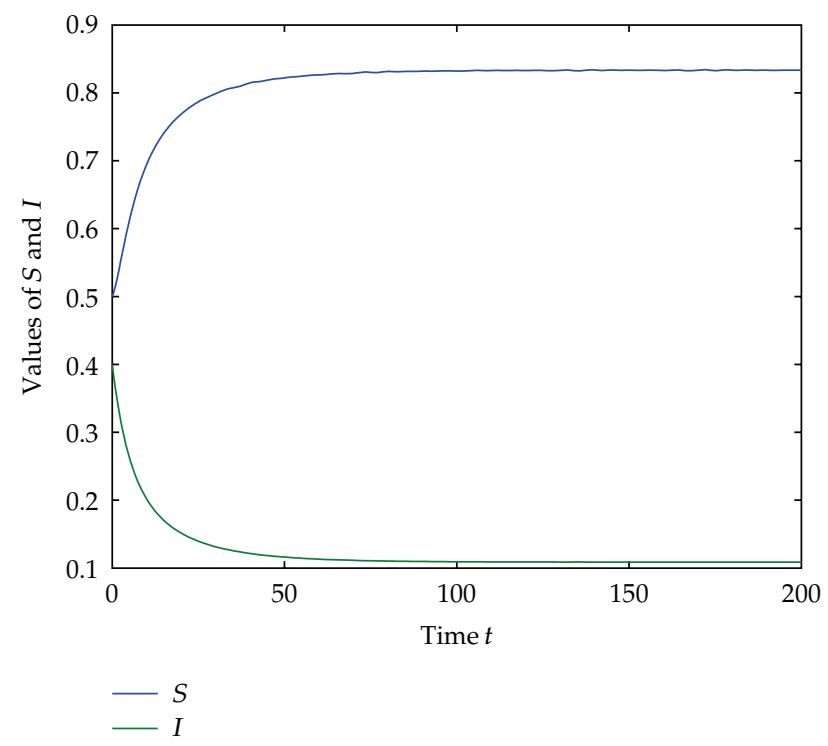

Figure 3: Evolutions of $S(t)$ and $I(t)$ for the system with $\beta=0.3, \delta=0.1, \alpha_{1}=0.2, \alpha_{2}=0.4, \gamma_{1}=0.1$, and $r_{2}=0.05$, provided $S(0)=0.5$ and $I(0)=0.4$.

From (2.2), it is easy to see that $R_{0}$ is increasing with $\beta$, and is decreasing with $\gamma_{1}, \gamma_{2}$, and $\delta$, respectively. This implies that prevention is more important than cure, and higher disconnecting rate from the Internet contributes to the suppression of virus diffusion.

As a consequence, it is highly recommended that one should regularly update the antivirus software even if their computer is not noticeably infected, and timely disconnect the computer from the Internet whenever this connection is unnecessary. Also, filtering and blocking suspicious messages with firewall is rewarding.

\section{Conclusions}

By considering the possibility that an infected computer becomes susceptible as well as the possibility that a susceptible computer becomes recovered, a new computer virus propagation model has been proposed. The dynamics of this model has been fully studied. On this basis, some effective measures for controlling the spread of computer viruses across the Internet have been posed.

\section{Acknowledgment}

The authors are greatly indebted to the anonymous reviewers for their valuable suggestions. This work is supported by the Natural Science Foundation of China (Grant no. 10771227), the Doctorate Foundation of Educational Ministry of China (Grant no. 20110191110022), and the Research Funds for the Central Universities (Grant nos. CDJXS12180007 and CDJXS10181130). 


\section{References}

[1] F. Cohen, "Computer virus: theory and experiments," Computers E Security, vol. 6, pp. 22-35, 1987.

[2] F. Cohen, A Short Course on Computer Viruses, John Wiley \& Sons, New York, NY, USA, 2nd edition, 1994.

[3] P. J. Denning, Computers Under Attack, Addison-Wesley, Reading, Mass, USA, 1990.

[4] J. R. C. Piqueira and V. O. Araujo, "A modified epidemiological model for computer viruses," Applied Mathematics and Computation, vol. 213, no. 2, pp. 355-360, 2009.

[5] P. S. Tippett, "The kinetics of computer viruses replication: a theory and preliminary survey, in: safe computing," in Proceedings of the 4th Annual Computer Virus and Security Conference, pp. 66-87, New York, NY, USA, March 1991.

[6] B. K. Mishra and S. K. Pandey, "Dynamic model of worms with vertical transmission in computer network," Applied Mathematics and Computation, vol. 217, no. 21, pp. 8438-8446, 2011.

[7] H. Yuan and G. Q. Chen, "Network virus-epidemic model with the point-to-group information propagation," Applied Mathematics and Computation, vol. 206, no. 1, pp. 357-367, 2008.

[8] B. K. Mishra and D. K. Saini, "SEIRS epidemic model with delay for transmission of malicious objects in computer network," Applied Mathematics and Computation, vol. 188, no. 2, pp. 1476-1482, 2007.

[9] X. Han and Q. L. Tan, "Dynamical behavior of computer virus on internet," Applied Mathematics and Computation, vol. 217, no. 6, pp. 2520-2526, 2010.

[10] A. Lahrouz, L. Omari, D. Kiouach, and A. Belmaâti, "Complete global stability for an SIRS epidemic model with generalized non-linear incidence and vaccination," Applied Mathematics and Computation, vol. 218, pp. 6519-6525, 2012.

[11] B. K. Mishra and N. Jha, "Fixed period of temporary immunity after run of anti-malicious software on computer nodes," Applied Mathematics and Computation, vol. 190, no. 2, pp. 1207-1212, 2007.

[12] B. K. Mishra and S. K. Pandey, "Fuzzy epidemic model for the transmission of worms in computer network," Nonlinear Analysis: Real World Applications, vol. 11, no. 5, pp. 4335-4341, 2010.

[13] J. Ren, X. Yang, L. X. Yang, Y. Xu, and F. Yang, "A delayed computer virus propagation model and its dynamics," Chaos, Solitons E Fractals, vol. 45, no. 1, pp. 74-79, 2012.

[14] J. Ren, X. Yang, Q. Zhu, L. X. Yang, and C. Zhang, "A novel computer virus model and its dynamics," Nonlinear Analysis: Real World Applications, vol. 13, no. 1, pp. 376-384, 2012.

[15] C. Vargas-De-León, "On the global stability of SIS, SIR and SIRS epidemic models with standard incidence," Chaos, Solitons \& Fractals, vol. 44, pp. 1106-1110, 2011.

[16] F. W. Wang, Y. K. Zhang, C. G. Wang, J. F. Ma, and S. J. Moon, “Stability analysis of a SEIQV epidemic model for rapid spreading worms," Computers \& Security, vol. 29, no. 4, pp. 410-418, 2010.

[17] B. K. Mishra and N. Jha, "SEIQRS model for the transmission of malicious objects in computer network," Applied Mathematical Modelling, vol. 34, no. 3, pp. 710-715, 2010. 


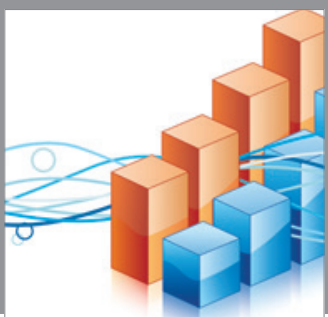

Advances in

Operations Research

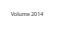

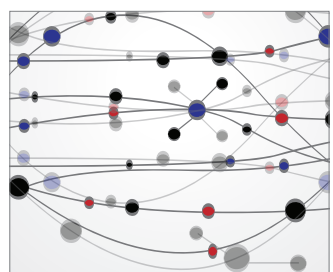

\section{The Scientific} World Journal
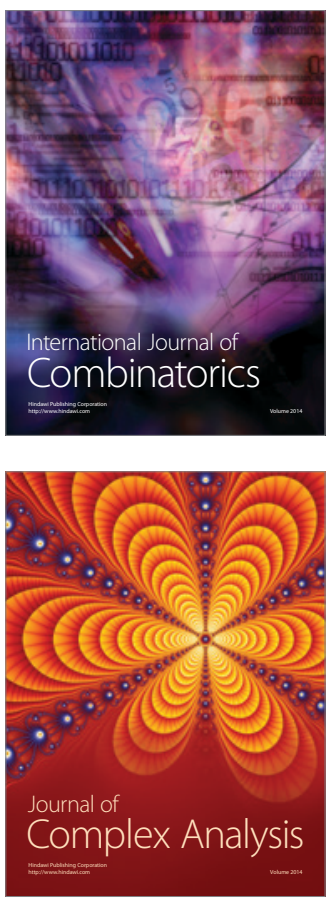

International Journal of

Mathematics and

Mathematical

Sciences
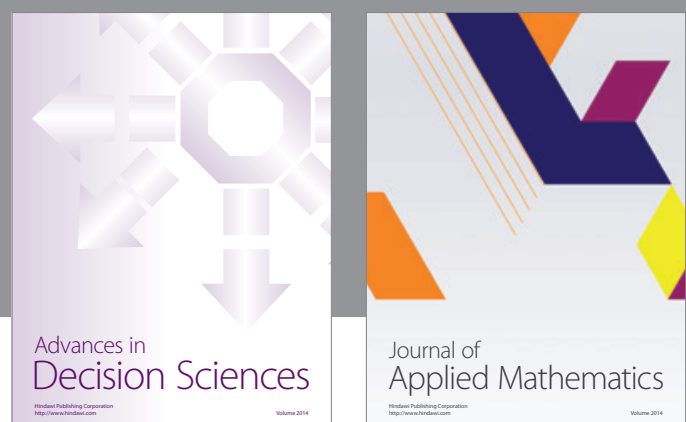

Journal of

Applied Mathematics
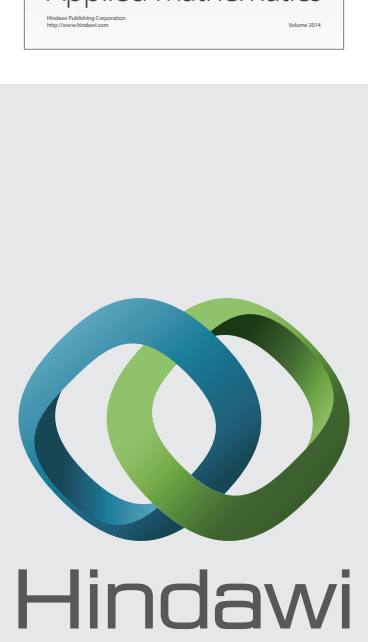

Submit your manuscripts at http://www.hindawi.com
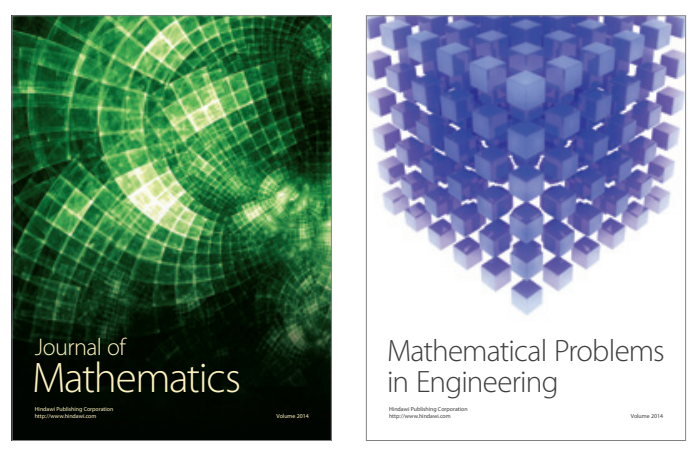

Mathematical Problems in Engineering
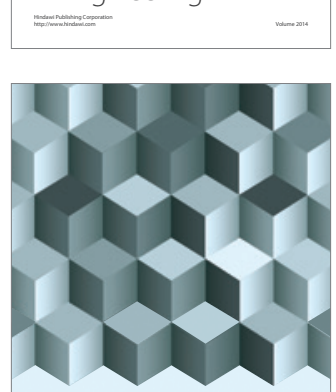

Journal of

Function Spaces
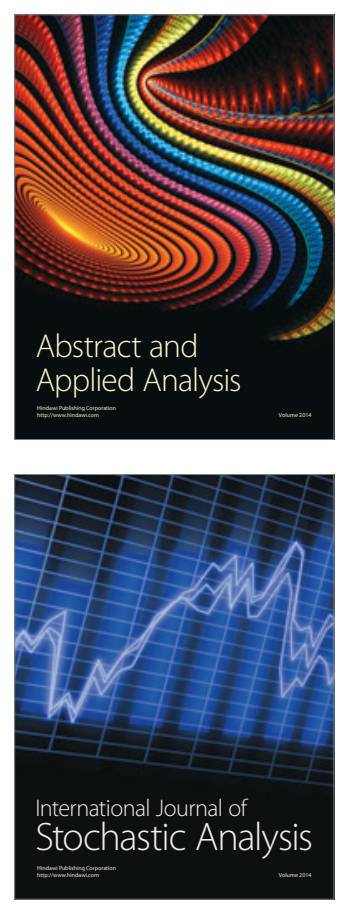

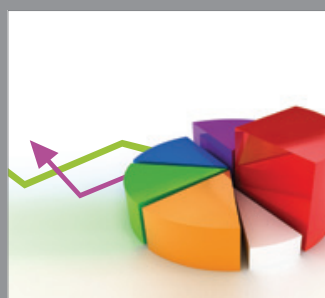

ournal of

Probability and Statistics

Promensencen
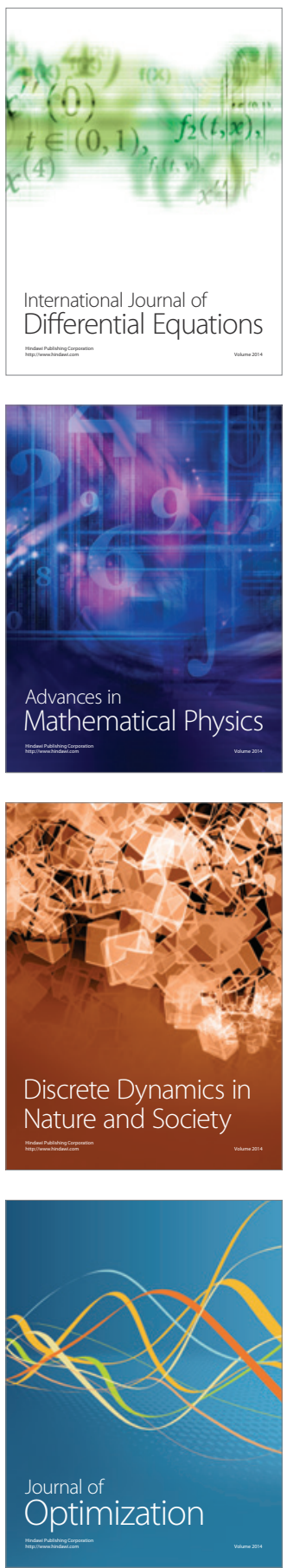